\title{
PLANEAMIENTO METROPOLITANO HETERODOXO Innovaciones institucionales e instrumentales del caso argentino
}

\author{
HETERODOX METROPOLITAN PLANNING \\ Institutional and instrumental innovations of the Argentine case
}

\author{
Elinbaum, Pablo \\ pabloelinbaum@gmail.com
}

\section{RESUMEN}

Los planes metropolitanos no pueden tipificarse, aunque en la bibliografía especializada prevalecen los ejemplos del "Norte" implementados durante el período desarrollista de postguerra. Más allá de las diferencias de contenido, estos planes comparten marcos estatales sostenidos por enormes orquestaciones de leyes y regulaciones. Pero, ¿qué sucede con las iniciativas de ordenación metropolitana que se desarrollan en los países que no cuentan con marcos institucionales formalizados, tal como se da en los países latinoamericanos? A partir de un estudio de caso comparado de dos planes metropolitanos argentinos, intentaremos echar luz sobre estas cuestiones, contribuyendo a los marcos de investigación y de la práctica. Considerando tres dimensiones analíticas -la institucional, la instrumental y la procesual-intentaremos definir la especificidad del planeamiento metropolitano en el contexto del Sur Global -como el argentino- y ofrecer un conjunto de lecciones que evidencien como las políticas públicas metropolitanas pueden ser operativas más allá del formalismo de los enfoques integrales.

Palabras clave: planeamiento metropolitano, marcos institucionales, innovación instrumental.

Bloque temático: Análisis y proyecto territorial.

\begin{abstract}
Metropolitan plans cannot be typified, although in the specialized literature prevails the examples of the "North", implemented during the post-war developmental period. Beyond content and scope differences, these plans share similar state frameworks supported by huge orchestrations of laws and regulations. But, what about the metropolitan planning initiatives that take place in countries that do not have formalized institutional frameworks, as is the case in Latin American countries? Based on a comparative case study of two Argentine metropolitan plans, we will try to shed light on these issues, contributing to the research and practice frameworks. Considering three analytical dimensions -institutional, instrumental and procedural- we will try to define the specificity of metropolitan planning in the context of the Global South, as in the case of Argentine, and offer a set of lessons that show how metropolitan public policies can be operational beyond formalist and integral approaches.
\end{abstract}

Keywords: metropolitan planning, institutional frameworks, instrumental innovation.

Topic: Analysis and territorial project. 


\section{Introducción}

A diferencia de los planes urbanos municipales -aquellos que cuentan con códigos y normas estandarizadas, resultante de una tradición administrativa de dos siglos-, los planes metropolitanos no pueden tipificarse, sino que deben redefinirse en cada ejercicio de planeamiento (Elinbaum, 2016). Sin embargo, en la bibliografía especializada aún prevalecen los paradigmas del "Norte": planes canonizados como el del Gran Londres (1944), el Schema Directour de París (1965) o el Plan de los Dedos de Copenhague (en danés, Fingerplanen) de 1947 -todos ellos implementados durante el período desarrollista de mediados del siglo XX. Mas allá de las tradiciones disciplinares de los distintos países europeos, ${ }^{1}$ los planes que "hicieron escuela" convergen en el contexto institucional de su implementación: marcos burocráticos sostenidos por enormes orquestaciones de leyes, regulaciones y proyectos estatales que demandaban las economías industriales fordistas.

Pese al auge de otros enfoques contemporáneos que surgen con la reestructuración económica postindustrial, como el planeamiento estratégico, el formalismo modernista permanece como el principal referente tecnológico en las investigaciones aplicadas y en el diseño de las políticas públicas metropolitanas. De este modo, el sesgo en la definición del planeamiento metropolitano como objeto de estudio ha estado claramente dominado, por un lado, en la aproximación racional-comprehensiva y, por otro, en las tradiciones de planeamiento "autorreferenciales" de la casuística del norte global que operan dentro del marco integrado de la Unión Europea, a menudo considerado como el ideal de articulación interadministrativa (Elinbaum \& Galland, 2018). Pero, ¿qué sucede con las iniciativas de ordenación metropolitana desarrolladas en los países latinoamericanos que no cuentan con marcos institucionales tan formalizados?, ¿es posible planificar por fuera - o sin la necesidad- de grandes marcos instrumentales y normativos integrados?, en definitiva, ¿qué podemos aprender de experiencias tan caracterizadas por la contingencia como las latinoamericanas?

El objetivo de esta comunicación apunta a contribuir a los marcos de investigación y de la práctica del planeamiento metropolitano, para mostrar cómo la operatividad y la innovación tecnológica no dependen solo de los marcos institucionales integrados o plenamente formalizados. En particular, apuntamos a conceptualizar la especificidad del planeamiento metropolitano en América Latina concibiéndolo como una práctica situada en su contexto -más que aislada o periférica. Con este fin, a partir de un estudio de caso comparado de dos planes metropolitanos argentinos (Rosario y Mendoza), exploraremos los marcos institucionales, el funcionamiento de los instrumentos y el proceso de elaboración del planeamiento, para identificar sus aspectos más operativos e innovadores. Pretendemos, en este sentido, mostrar cómo este tipo de planeamiento metropolitano, que denominaremos "heterodoxo", en base al modelo no-euclidiano de John Friedmann (1993) y a la "ontología escalar plana" postulada por Sallie Marston et. al (2005), disminuye la burocracia y aumenta la operatividad, en la medida que permite adecuar los instrumentos y la gestión a las cambiantes coyunturas políticas e institucionales, al armado ad-hoc de equipos técnicos, y a la oferta de múltiples fuentes de financiamiento.

Esta investigación parte de un enfoque cualitativo y se apoya en la técnica del estudio de caso comparado. El planeamiento en las áreas metropolitanas de Rosario y Mendoza fue seleccionado en base a cuatro condiciones tipológicas: (1) ambos casos son coetáneos, (2) se enmarcan en el Programa de Desarrollo de Áreas Metropolitanas del Interior (DAMI), ${ }^{2}$ (3) presentan instituciones específicas para la coordinación

\footnotetext{
${ }^{1}$ Según Nadin y Stead (2008), dentro de Europa, podemos distinguir cuatro grandes tradiciones de planeamiento: Comprehensivointegrado, Urbanístico, Económico-regional y Regulación del uso del suelo. Dentro de estas tradiciones existen, obviamente, matices regionales e históricos. Así, dentro del estilo "Urbanístico" podemos distinguir el enfoque italiano del español que, a su vez, tiene su propia generación de planes: de aprovechamiento edilicio, remediales y morfológicos (Font, 2000). Asimismo, cada país europeo muestra combinaciones de las "tradiciones ideales". Por ejemplo, España se considera un país con una tradición urbanística y, al mismo tiempo, se lo asocia a la tradición de la regulación del uso del suelo, por el legalismo que caracteriza su estatalidad (Farinós, 2007).

${ }^{2}$ El Programa DAMI -dependiente del Ministerio del Interior, Obras Públicas y Vivienda- tiene como propósito contribuir a mejorar el funcionamiento de los servicios en las áreas metropolitanas del interior (AMI) y poner en marcha modalidades innovadoras de gestión para
} 
metropolitana, y (4) superan el tradicional enfoque sectorial de las políticas públicas metropolitanas. Para el análisis de los casos se recurrió a fuentes secundarias como la documentación de los planes, las leyes y normas vigentes en cada contexto, la bibliografía especializada sobre los casos, y diferentes fuentes públicas como las que se publican en los portales oficiales de los organismos estatales. Asimismo, nos hemos apoyado en una serie de entrevistas semi-estructuradas realizadas a las coordinadoras técnicas en cada caso.

El artículo se estructura en cuatro partes. Después de la introducción, en el segundo apartado se explica el marco conceptual y metodológico para el análisis empírico, a partir de tres dimensiones: la institucional, la instrumental y la procesal. En el tercer apartado se desarrollan los estudios de caso. Por último, se cruzan y discuten los resultados y se ofrecen algunas observaciones convergentes en los casos, sugiriendo posibles caminos para las investigaciones futuras.

\section{Las tres dimensiones del planeamiento metropolitano}

¿Cómo se analizan los planes metropolitanos? o, siendo más generales en cuanto al objeto que estamos tratando, cabría preguntar: ¿cómo se estudian las políticas públicas urbanas? A partir de la revisión bibliográfica, recopilamos las principales contribuciones de diferentes autores que han estudiado el alcance y el contenido, entre otras características básicas, de los planes urbanos y de ordenamiento territorial. Yendo más lejos, en esta sección se sugiere una serie de dimensiones y variables analíticas que apuntan evaluar el diseño y la implementación de los planes metropolitanos contemporáneos. Partiendo del estudio canónico sobre la doctrina de planificación holandesa, escrito por Faludi y Van der Valk (1994), consideraremos dos tipos básicos de planificación, la tecnocrática y la sociocrática, asociados respectivamente con formas de intervención proyectuales y estratégicas. Sobre la base de esta distinción, con el objetivo de explicar los aspectos innovadores de los planes metropolitanos, se proponen tres dimensiones analíticas -el contexto institucional, el contenido instrumental y el proceso de planificación (ver Tabla 1).

El contexto institucional de los planes metropolitanos se caracteriza esencialmente por el marco legal de un determinado país y por los organismos que participan en el diseño e implementación de los planes (Newman \& Thornley, 1996; CEC, 1997). En este trabajo, se opta por distinguirlos en marcos "conformativos" y "movilizadores", ${ }^{3}$ tal como lo define Janin Rivolin (2008). Desde esta perspectiva, se considera que las políticas y modelos metropolitanos reproducen esencialmente los sistemas de planeamiento a través de los cuales se desarrollan. Así, podemos distinguir un enfoque más tradicional y amplio, que aspira a "conformar" proyectos unitarios en una estrategia integral, en la cual los actores están obligados a participar; y otro menos institucionalizado, que promueve aquellos proyectos capaces de "movilizar" una estrategia desestructurada donde los actores están invitados a participar" (Janin Ravolin, op. cit., p. 167). De este modo, las instituciones involucradas podrían estar "conformadas" por una institución monolítica (única) o "movilizadas" por una coalición de actores involucrados en el proceso de elaboración del plan (Faludi \& Van der Valk, op. cit.; Roodbol-Mekkes et al., 2012; Van Duinen, 2013).

En cuanto al procesos de planeamiento, la programación de los objetivos y los escenarios, éstos se relacionan con lo que Faludi (2000) denomina "elemento temporal". Es a través de estos elementos que los planes pueden concebirse de forma incremental (como pasos encadenados para alcanzar el resultado previsto en el horizonte del plan) u opcional, cuando se prevén caninos alternativos de implementación (p. 303). Además, el proceso de planificación depende en buena medida de los papeles que adoptan los planificadores que, al mismo tiempo, caracterizan el estilo de planeamiento. Para simplificar su definición, tal como las describen Faludi y Van der Valk (1994), los clasificaremos a partir de dos enfoques genéricos -el tecnocrático y el participativo-, haciendo la salvedad de que, en la práctica, los estilos no son puros, sino que tienden a combinarse. Finalmente, los

la ejecución de proyectos y la prestación de servicios cuyo desempeño eficiente requiera de la concurrencia de dos o más jurisdicciones territoriales (nacional, provincial y/o municipal).

${ }^{3}$ Creemos que son éstos los términos que mejor se ajusten al concepto original en inglés: conformative y performative. 
procesos de planificación pueden evaluarse a partir del desempeño del planeamiento, dependiendo de si este se evalúa de forma continua y sistemática o de forma eventual, en función de sus componentes e indicadores concretos (Faludi, op. cit., p. 310).

\begin{tabular}{lll} 
Dimensión & Variable & Parámetro \\
\hline $\begin{array}{lll}\text { Contexto } \\
\text { Institucional }\end{array}$ & Marco legal & Conformativo vs. movilizador \\
\cline { 2 - 3 } $\begin{array}{l}\text { Contenido } \\
\text { Instrumental }\end{array}$ & Instituciones involucradas & Monolítico vs. Coalición \\
\cline { 2 - 3 } & Fontenido temático & General vs. Selectivo \\
\hline $\begin{array}{l}\text { Proceso de } \\
\text { Planeamiento }\end{array}$ & Escenarios & Vinculante vs. Indicativo \\
\cline { 2 - 3 } & Papel de los técnicos & Incrementales (fases) vs. Opcional \\
\hline
\end{tabular}

Tabla 1. Dimensiones, variables y parámetros claves del planeamiento metropolitano. Fuente: Adaptado de las categorías propuestas por Faludi and Van der Valk (1994)

\section{El planeamiento metropolitano en el Gran Rosario. La mirada desde abajo}

El Área Metropolitana de Rosario (AMR) comprende un conjunto dinámico y heterogéneo de localidades ubicadas al sur de la provincia de Santa Fe, atadas por su interdependencia funcional, y pertenecientes a un mismo proceso de conformación territorial, en el que se identifica un conjunto de problemáticas comunes (déficit de viviendas, contaminación por el uso de agroquímicos, congestión del transporte de carga, solo por mencionar los más acuciantes). Dicho proceso ha sido abordado en distintas instancias, reconociendo la dinámica singular que le imprime Rosario, la ciudad central, pero, también, el rol que juega en su consolidación el conjunto de localidades que la rodean, conformando una estructura tentacular que se extiende desde la vera del río Paraná hasta más allá de la autovía A012, sobre el frente pampeano; y en sentido norte sur, como un cordón lineal recostado sobre la costa (Fig. 1).

Al margen de la breve experiencia de la Prefectura del Gran Rosario (1969-1976), clausurada durante la última dictadura militar, la institucionalidad metropolitana se consolida en la primera década del siglo XXI a partir del proceso de planeamiento estratégico sostenido gracias a la continuidad del Partido Socialista en Rosario y luego en la Provincia. Experiencias como el Plan Estratégico Provincial (PET-2030) y Plan Estratégico Rosario Metropolitana (PERM+10), permitieron que, en 2012, se instituya en el Ente de Coordinación Metropolitana de Rosario (ECOM). El ECOM nuclea de forma voluntaria a 26 localidades organizadas en un consejo de gobierno formado por todos los intendentes; ${ }^{4}$ un directorio constituido por cinco jefes de gobierno que van rotando; y

\footnotetext{
${ }^{4}$ El Área Metropolitana de Rosario (AMR) está conformada por los municipios de: Acebal, Álvarez, Alvear, Andino, Arroyo Seco, Capitán Bermúdez, Coronel Domínguez, Fighiera, Funes, General Lagos, Granadero Baigorria, Ibarlucea, Pérez, Piñero, Pueblo Esther, Ricardone, Rosario, San Lorenzo, Soldini, Villa Gobernador y Zavalla. El AMR concentra más de 1.400 .000 habitantes que residen en un espacio que ocupa cerca de $2.200 \mathrm{~km}^{2}$ de superficie. En ese territorio vive el $40 \%$ de la población de la Provincia de Santa Fe y el 3,1\% del país. Sólo el 18\% de ese suelo (24.000 hectáreas) está ocupado por los núcleos urbanos y suburbanos. El 82\% restante (aproximadamente 112.000 hectáreas) corresponden a suelo rural, valor que evidencia el peso que adquiere la ruralidad en la conformación del área.
} 
una presidencia reservada para el intendente de Rosario. En 2016, la Ley de Áreas Metropolitanas confirmó este organigrama, reforzando aún más la autarquía del ECOM.

Pese a su institucionalidad formal, en la práctica, el ECOM funciona como una coalición de actores cuya cohesión nunca está garantizada. Para lograr la horizontalidad entre los intendentes fue necesario enfocar la cooperación intermunicipal exclusivamente a través de discusiones técnicas $y$, sobre todo, de proyectos concretos. En este sentido, cabe destacar el rol la secretaría ejecutiva del ECOM, la "Oficina Metropolitana", encargada de la gestión del planeamiento urbano y de los proyectos de alcance supralocal, como, por ejemplo, la planta de Gestión Integral de Residuos Sólidos Urbanos ubicada en Villa Gobernador Gálvez y la reconversión de la Ruta 21 en la avenida interurbana actualmente en obra (Fig. 2). El trabajo cotidiano en el apoyo técnico fue perfilando al ECOM como un mediador entre las localidades y la provincia. Este papel, sin embargo, tiene un doble enfoque instrumental y político. Uno desde abajo, horizontal, que permite incorporar las cuestiones locales en las políticas sectoriales provinciales, de forma integrada y sistematizada. Y otro enfoque desde arriba, vertical, que alinea a los intendentes metropolitanos a las políticas del gobierno provincial, en la medida que éste tiende a concentrar y distribuir el presupuesto en obras públicas a través del ECOM.

Pese a los diferentes ejercicios de restructuración del espacio estatal y a las leyes aprobadas, la integridad institucional del ECOM depende, sobre todo, de un trabajo más afectivo que normativo, basado en nutrir la confianza y el sentimiento de pertenencia institucional. La actual coyuntura política se sostiene a partir de dos estrategias. La primera apunta a arraigar la conciencia metropolitana a través de un discurso que sea capaz de explicar de manera didáctica, persuasiva y convincente los criterios y objetivos para el desarrollo metropolitano. La segunda estrategia, radica en garantizar la horizontalidad de los intendentes en el marco del consejo de gobierno del ECOM. El énfasis puntilloso en esta última estrategia es tal que obligó a recortar el municipio de Rosario del mapa metropolitano, liberando los efectos de su macrocefalia urbana e institucional.

Partiendo de un enfoque urbanístico de la ordenación territorial, propio de los arquitectos, el ECOM dirige sus objetivos a partir de tres grandes temas: espacios libres, movilidad y urbanización. Pese al carácter genérico de estos temas, las Directrices de Ordenación Territorial (DOT) formuladas en 2014 hacen hincapié en aspectos específicamente metropolitanos, evitando la superposición con los planes urbanos locales. Desde esta perspectiva, los espacios libres se centran en el tratamiento del suelo periurbano, la movilidad se enfoca desde el diseño de las vías interurbanas y la logística, y las políticas de urbanización se plantean delimitando áreas de expansión de la planta urbana que demanden proyectos específicos y argumentos que trasciendan la escala local. 


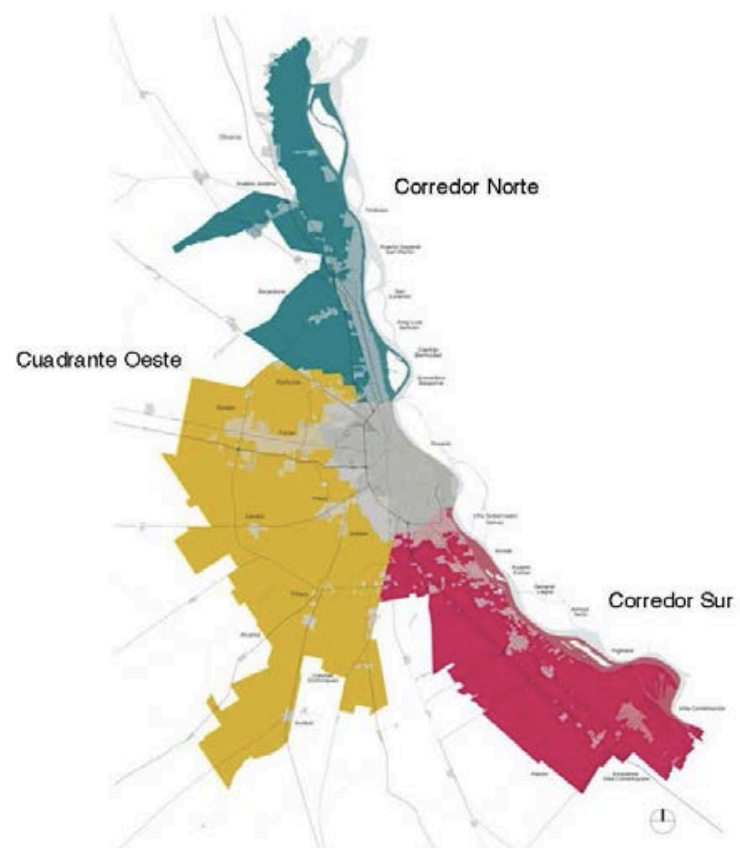

FIG. 1. Sub-sistemas urbanos del área metropolitana de Rosario. Fuente: Archivo del ECOM, 2018 

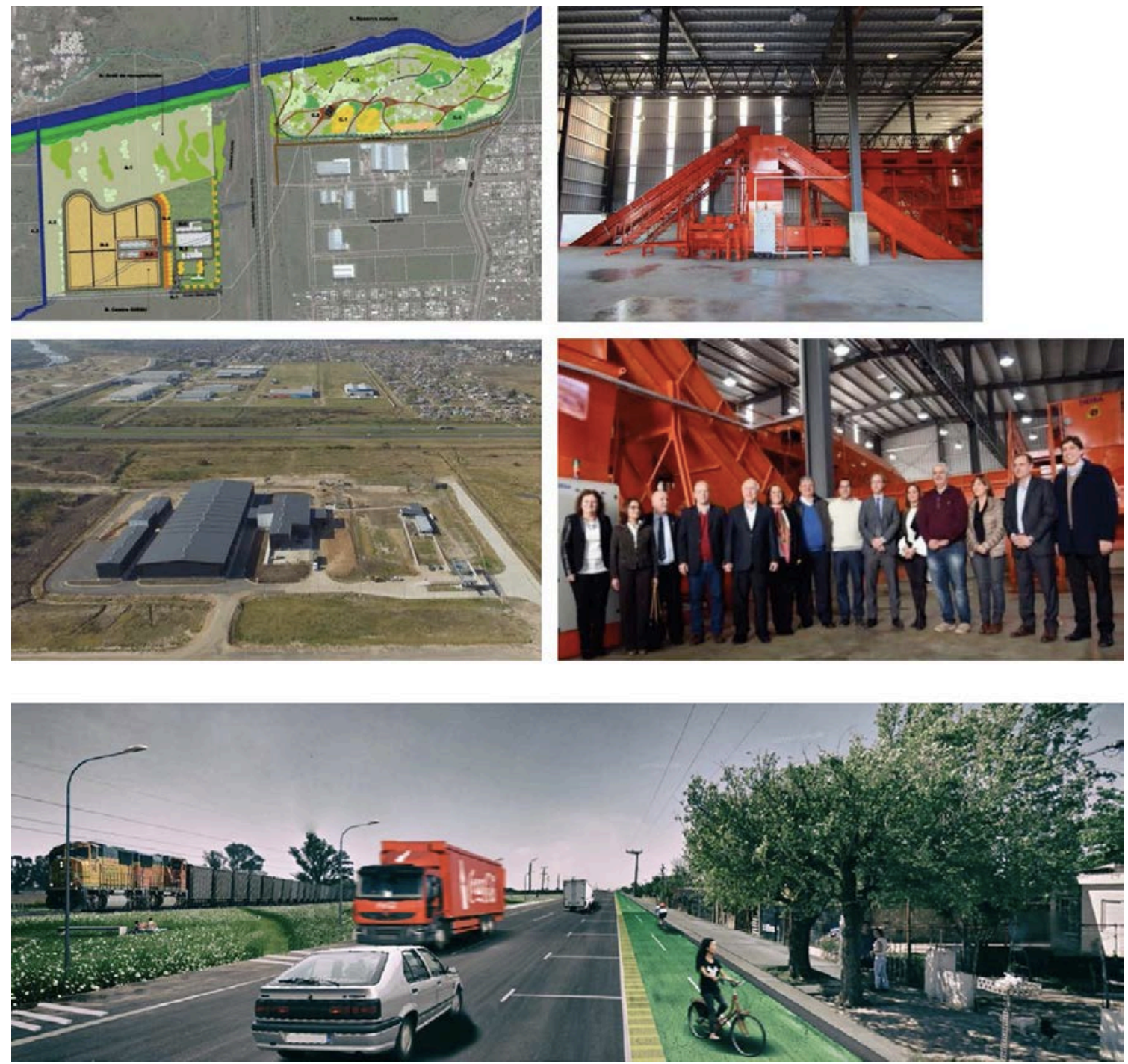

FIG. 2. Dos de las obras de alcance supralocal gestionadas y ejecutadas a través del ECOM. Arriba: Centro GIRSU. Abajo: Proyecto de reconversión de la Ruta 21. Fuente: Archivo del ECOM.

Pese a la racionalidad de los criterios que avalan el desarrollo supralocal, la delicada coyuntura entre los intendentes dejó en claro que transformar las DOT en un plan metropolitano sería contraproducente para la incipiente institucionalidad del ECOM. Por eso, se optó por implementarlas desde abajo, a partir de la redacción de los planes urbanos para cada localidad, previendo que contengan las cuestiones metropolitanas (Fig. 3). De este modo, la sumatoria de los planes locales resultaría "de facto" en el plan metropolitano, sin necesidad de imponerlo. Aun así, si bien la normativa urbanística es competencia de los gobiernos locales, el ECOM se reserva tres temas estratégicos para implementarlos mediante leyes provinciales sectoriales. Estas son: la protección de los frentes fluviales, el respaldo jurídico de las rondas y arterias interurbanas, y la fijación del suelo urbanizable. En relación a esto último, la reserva de suelo se concibe con un enfoque más estratégico que normativo. No se trata de imponer la forma de la expansión en cada localidad, sino de que los intendentes reaccionen -realizando un proyecto oportuno para el municipio- antes de que lo hagan los promotores. 


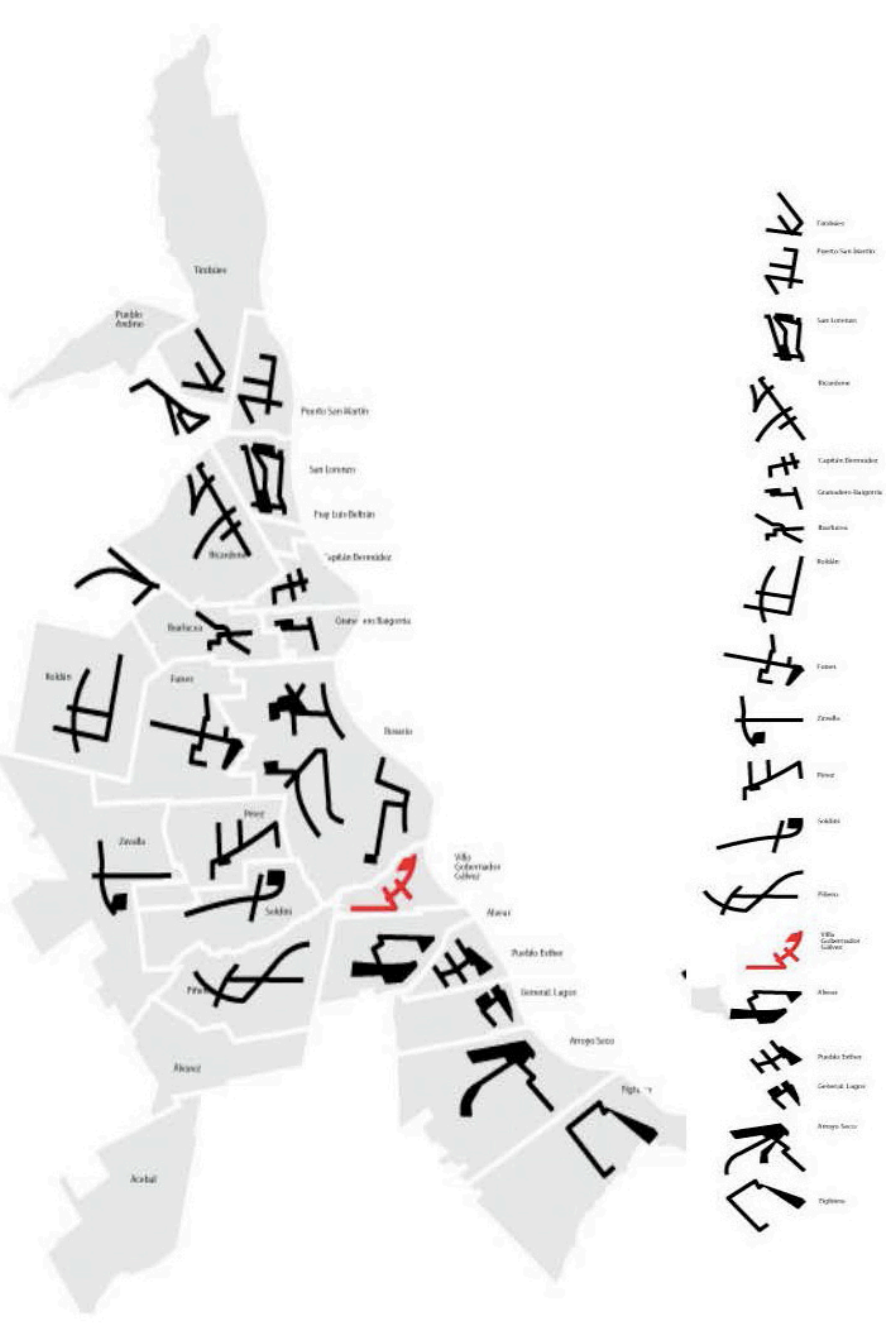

FIG.3. Plano de pictogramas que representan la parte del proyecto metropolitano que le toca a cada localidad, enfatizando no solo la horizontalidad sino el carácter imprescindible en la conformación de un único proyecto metropolitano. Fuente: Archivo del ECOM, 2016.

Si bien, las DOT están pensadas para la acción y para la gestión urbanística, hasta la fecha el ECOM no ha fijado un programa de intervenciones, ni ha definido escenarios de desarrollo. La articulación de las DOT con las agendas municipales demanda que primero se aprueben todos los planes urbanos locales. Este proceso aún en marcha contempla instancias participativas en dos niveles: el del consejo de intendentes y el de los talleres ciudadanos que se realizan durante la elaboración de los planes urbanos locales. Los talleres son eventos sociales de gran relevancia que, por la escala menuda de los pueblos metropolitanos, suelen marcan un giro en su historia: por primera vez se discuten los problemas urbanos de forma integral y el futuro de su ciudad a largo plazo. Los talleres también se aprovechan para divulgar los aspectos supralocales de cada plan local, anticipando el nuevo régimen metropolitano.

Es en este punto donde el caso del ECOM evidencia cómo la escala indefinida de lo metropolitano demanda un reequilibrio continuo entre las formas y los procesos. Veamos a continuación cómo se equilibran estos términos en el caso del área metropolitana de Mendoza. 


\section{El planeamiento metropolitano en el Gran Mendoza. La mirada desde arriba}

Con casi un millón de habitantes, el área metropolitana de Mendoza es la principal conurbación del oeste argentino y la cuarta del país. Está compuesta por los municipios de Las Heras, Guaymallén, Godoy Cruz, Maipú, Luján de Cuyo y Ciudad de Mendoza, concentrando más del $65 \%$ de la población de la provincia y ocupando $168 \mathrm{~km}^{2}$ extendidos de forma casi regular hasta las estribaciones de la cordillera de los Andes que impiden el crecimiento. Sin embargo, por su localización estratégica en el sistema nacional de ciudades y el corredor Bioceánico, el área metropolitana de Mendoza es un importante prestador de bienes y servicios tanto a nivel local y regional como internacional. En los últimos cuarenta años ha experimentado un crecimiento demográfico, urbano y económico que provocó transformaciones en su configuración como metrópolis. Este proceso, condicionado por la vulnerabilidad sísmica y acompañado del enfoque sectorial de la planificación, resultó en un modelo de ciudad dispersa y de baja densidad que comienza a manifestar sus efectos negativos (expansión urbana descontrolada, avance hacia tierras de cultivo, exposición de la población a amenazas naturales, deposición final de residuos sólidos urbanos, dificultad con el servicio de transporte público de pasajeros e ineficiencia en la gestión de los recursos naturales).

Tras la experiencia del planeamiento estratégico (Mendoza 2010 y 2030) con un claro protagonismo del sector privado, el gobierno provincial inició el proceso de planificación que se formaliza en 2017 con la aprobación del Plan Provincial de Ordenamiento Territorial (PPOT). La sanción de la Ley provincial Nº899 hizo que Mendoza sea pionera en materia de planeamiento supralocal, en el contexto argentino. Como parte de este proceso, que duró casi diez años, en diciembre del 2015 se crea UNICIPIO, un Consejo de Políticas Públicas conformado por los siete municipios del área metropolitana y la Provincia, entrelazando fáctica y empíricamente el proyecto metropolitano con los objetivos del PPOT.

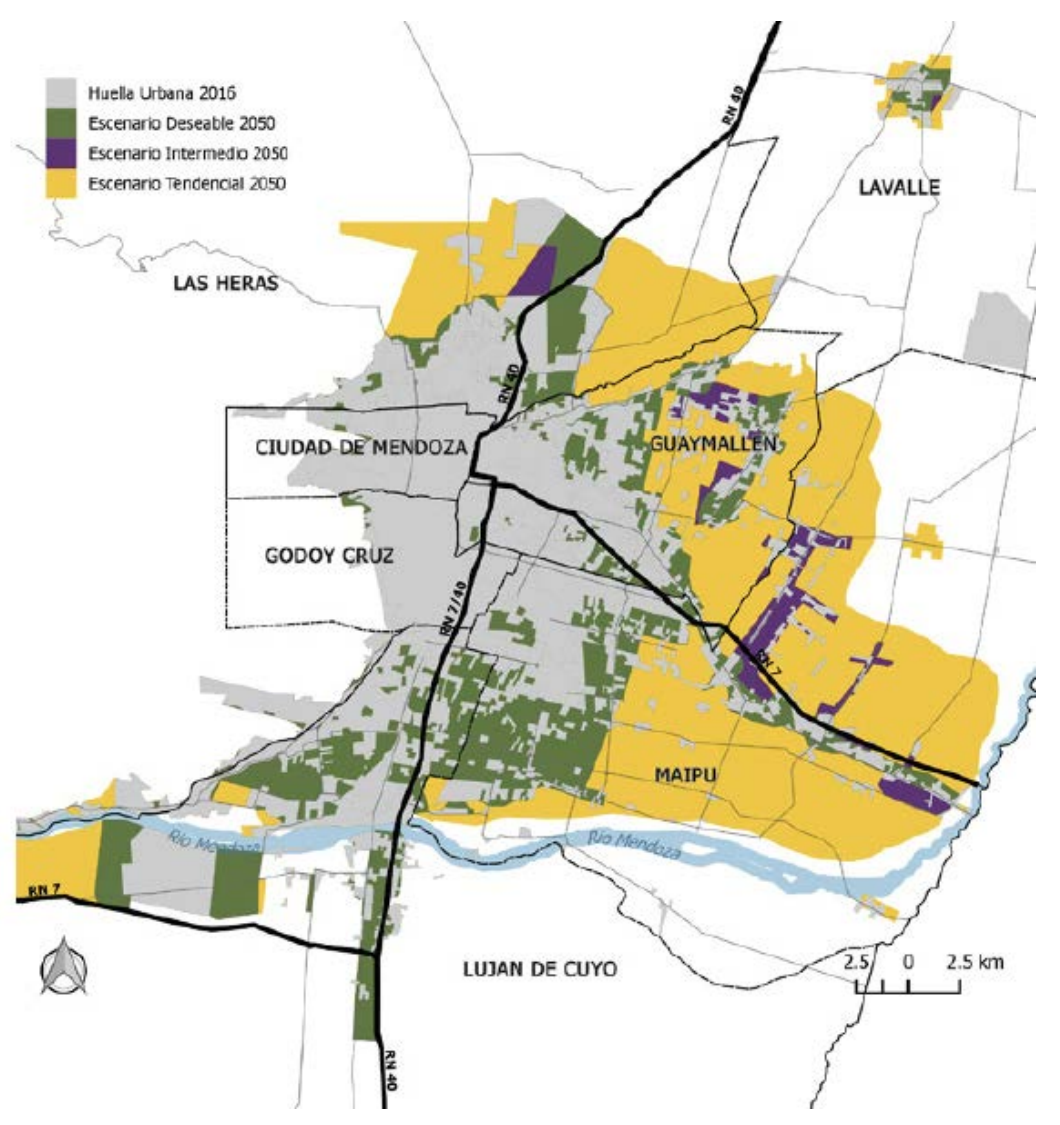

FIG. 4. Escenarios de desarrollo urbano. Fuente: Plan de Acción. UNICIPIO, 2018. 
El organigrama de UNICIPIO está conformado por un presidente (el gobernador), un coordinador (de la Secretaría de Ambiente y Ordenamiento Territorial), un representante (de la Agencia de Ordenamiento Territorial), y los siete intendentes y técnicos municipales. Pese a los vínculos de subsidiariedad administrativa con respecto a los gobiernos locales, UNICIPIO aún tiene un estatus de programa dentro del Estado provincial. Por esta razón, cuenta con un peso institucional limitado en relación a otras secretarias y ministerios. Si bien la "cuestión metropolitana" decae en UNICIPIO casi de forma tácita, el aval político del gobierno provincial ha sido suficiente como para realizar proyectos sofisticados como el Censo informático del arbolado público, armar equipos técnicos ad hoc, obtener recursos de diferentes fuentes y niveles administrativos (solo el $30 \%$ de la financiación de UNICIPIO proviene de la provincia), y fortalecer la coalición de todos los intendentes metropolitanos. Así, paradójicamente, la liviandad institucional ha permitido aumentar la autonomía de la gestión en relación a los objetivos para el desarrollo metropolitano.

Al depender de una secretaría provincial, podría decirse que UNICIPIO adopta un enfoque territorial "desde arriba" que diluye el peso institucional que históricamente tuvo el municipio de Mendoza, la ciudad capital, hoy completamente subsumida en la conurbación. Sin embargo, al principio, no todos los intendentes percibieron que UNICIPIO podía ser una institución horizontal. Hizo falta enfatizar los aspectos técnicos en las discusiones, para que todos los municipios se incorporen a la mesa metropolitana, incluyendo aquellos, como Maipú, de signo político opuesto al del gobierno provincial. La primacía de las cuestiones técnicas también permitió ajustar la delimitación del área metropolitana, incluyendo el municipio de Lavalle (omitido en el primer programa del DAMI); una localidad periférica a las dinámicas metropolitanas, pero fundamental desde el punto de vista de la red hídrica, en la medida que es a través de ella que se abastece de agua a toda el área metropolitana.

En cuanto al contenido de las políticas del UNICIPIO, podría decirse que se pasó del enfoque sectorial del primer programa DAMI, centrado en el problema de la seguridad, a otro más integral. A través del segundo programa, UNICIPIO redactó el Plan de Acción "Área Metropolitana de Mendoza Sostenible", enmarcado en la metodología de planeamiento CES (Ciudades Emergentes Sostenibles) del BID, que es el ente que financió el plan. Se trata de un protocolo estandarizado y estructurado en tres etapas (diagnostico, priorización y plan de acción) y en tres "pilares" (medio natural, socio-económico y fiscal, y urbano-funcional) operativizados por una serie de indicadores, previamente establecidos, que indican qué es sostenible y qué no.

La etapa de priorización, quizás la más original desde el punto de vista metodológico, se basa en la selección de los 14 (de 27) subtemas más problemáticos, "filtrados" por una encuesta de opinión pública y por la mirada interdisciplinar de los especialistas (ver fig. 5). Los temas seleccionados se desagregan en líneas de acción concretas como la gestión de los residuos, la promoción de clusters de innovación y la seguridad ciudadana, entre otros. Pese al carácter estandarizado de la metodología CES, el Plan de Acción también se articula con las políticas territorialmente situadas del PPOT, entre las que cabe destacar la revalorización del patrimonio cultural, la gestión del riesgo sísmico y la regularización de las actividades ubicadas al pie de monte. Se trata pues de un sofisticado ejercicio de concurrencia del planeamiento en múltiples escalas, donde el nivel metropolitano funciona como un catalizador que sintetiza diversas políticas, metodologías e instrumentos (ver fig. 6).

Cabe señalar que, pese al carácter comprehensivo de la metodología CES, las líneas de acción no son normativas, sino que están enfocadas en escenarios de desarrollo (actual, tendencial, deseable e intermedio), y en etapas y montos de inversión a corto, mediano y largo plazo (que también son orientativos). No obstante, más allá del carácter indicativo del Plan de Acción, UNICIPIO instauró en la gestión un protocolo vinculante para regular el proceso de urbanización. A partir de esta formalidad, los municipios metropolitanos deben someter sus expectativas y proyectos de expansión urbana a una instancia consultiva por parte de la Secretaría de Ambiente y Ordenamiento Territorial, que puede aprobarlos o no.

Asimismo, el proceso de elaboración del Plan, acorde a la metodología del BID, se apoyó en una encuesta 
ciudadana. Si bien esta fue la única instancia participativa, una vez redactado el Plan, la discusión de las líneas de acción continúa en las reuniones que coordina UNICIPIO de forma periódica con todas las localidades. Se trata de una "mesa metropolitana" en la que prevalece el perfil técnico, en la medida que los intendentes no suelen asistir. Si bien ausencia de los intendentes le quita peso político a la ordenación territorial, el papel protagonista de los planificadores ha sido clave para promover la consciencia sobre "lo metropolitano", y para clarificar los beneficios de la "cooperación" -y las limitaciones de la "coordinación", por ejemplo, cuando los municipios con más peso político, como la ciudad de Mendoza, ven limitada su histórica autonomía.

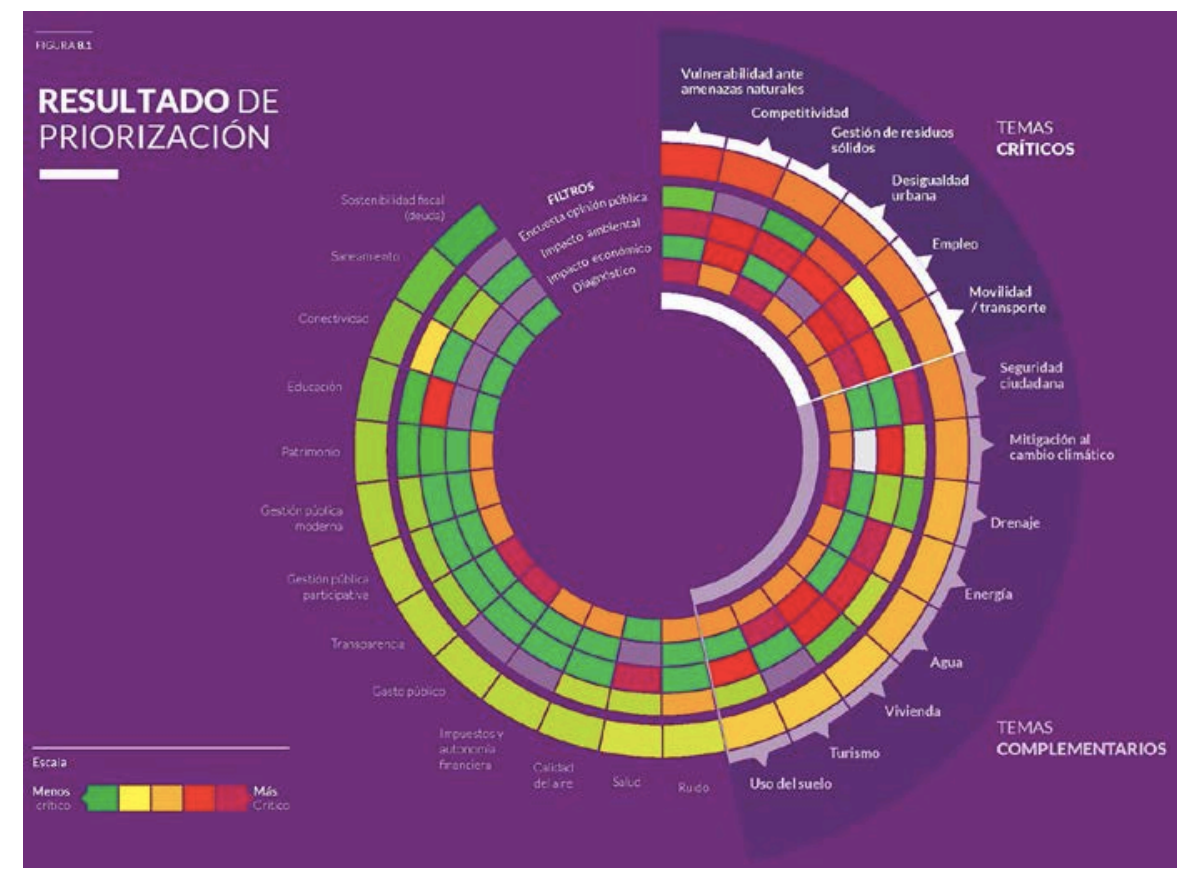

FIG. 5. Mecanismo de "filtros" para la priorización de temas y problemas. Fuente: Plan de Acción. UNICIPIO, 2018.

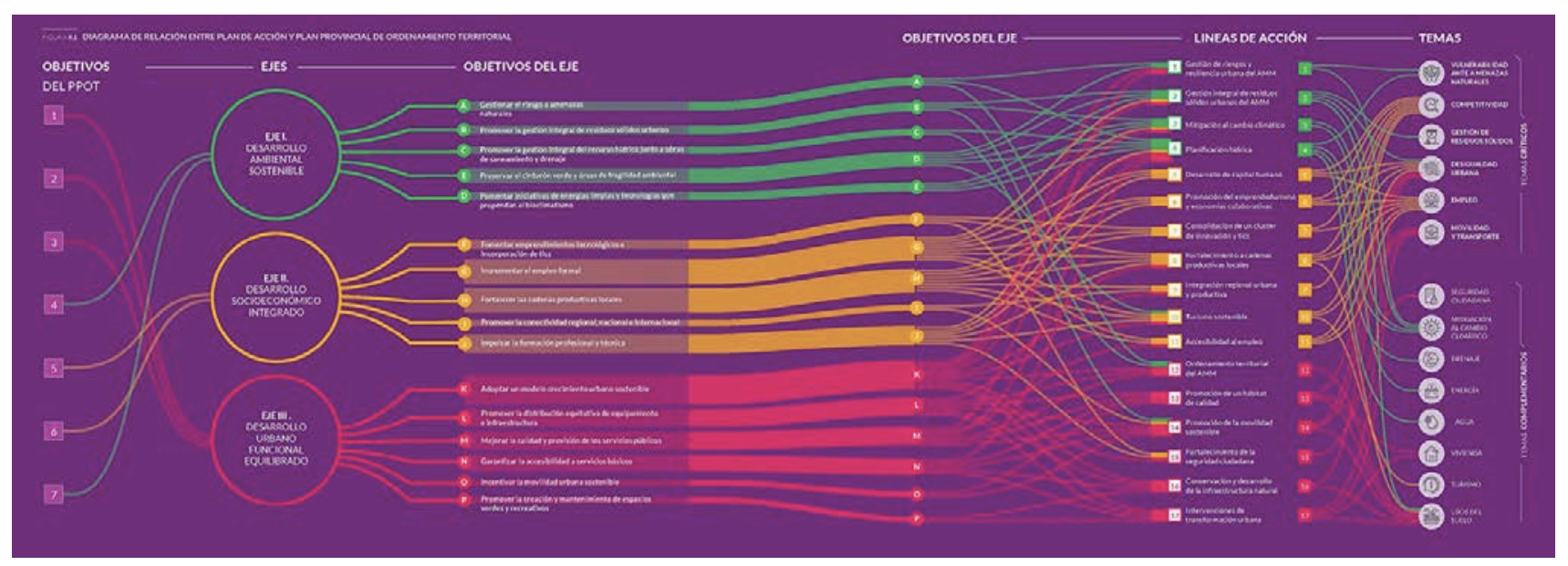

FIG. 6. Diagrama de relación entre el plan de acción y el plan provincial territorial. Fuente: Plan de Acción. UNICIPIO, 2018

\section{Colusiones}


Esta ponencia pretende contribuir a los marcos de investigación del planeamiento metropolitano en América Latina concibiéndolo como una práctica situada en su contexto. Es en este sentido que intentamos redefinirlo como objeto de estudio, superando el sesgo de las aproximaciones modernistas del período fordista y la idealización de las tradiciones de planeamiento "autorreferenciales" de la casuística del Norte Global. A parir de la comparación de dos experiencias notables del reciente planeamiento metropolitano argentino (Rosario y Mendoza), retomamos los interrogantes planteados en la introducción para indagar en su especificidad. El estudio de los casos enfatizó la importancia de la flexibilidad instrumental e institucional como condición estratégica para adaptar las políticas plurimunicipales a territorios y coyunturas específicas. En este sentido, las tres dimensiones analíticas de partida -la institucional, la instrumental y la procesal-, permiten concluir con tres argumentos concurrentes:

(1) Los entes metropolitanos tienen un enfoque institucional ambivalente. Por un lado, se conforman desde "desde arriba", dentro del Estado provincial y atados al marco integrado de las leyes y objetivos del ordenamiento territorial. Así, la relación entre los intendentes adquiere la forma de coalición a favor -o en oposición- al gobierno provincial de turno. El segundo tipo de encuadre se da "desde abajo", a partir de la organización voluntaria de los intendentes metropolitanos. Con el tiempo, estás asociaciones cristalizan en instituciones monolíticas, "casi" autárquicas, que nunca se independizan del apoyo del gobierno provincial o del de la ciudad capital. En los dos casos, la delicada unidad institucional depende de dos estrategias. La primera pretende arraigar la conciencia metropolitana de los intendentes a través de discursos persuasivos que sean capaces de reinterpretar los problemas locales en clave regional. Y la segunda apunta a reducir los conflictos políticos, centrando las discusiones exclusivamente en cuestiones técnicas y proyectos concretos. En definitiva, la institucionalidad de los entes metropolitanos no depende tanto de las leyes o de los acuerdos formales, sino de su capacidad para estabilizar la coyuntura entre los actores.

(2) El funcionamiento coyuntural de las instituciones plurimunicipales convierte al "nivel" metropolitano un catalizador que debe sintetizar políticas, instrumentos y metodologías tan disímiles como la doctrina del desarrollo sostenible, el enfoque situado del ordenamiento territorial, o la aproximación arquitectónica de los urbanistas. En cualquier caso, se trata de evitar la superposición con los planes locales, enfocando las políticas metropolitanas desde problemáticas específicas como, por ejemplo, el diseño de las vías regionales, la localización de las plantas de tratamiento de residuos o el transporte interurbano. Debido a la complejidad de estos temas, los instrumentos de planeamiento supralocal no suelen ser obligatorios. Aún así, los entes metropolitanos implementan distintos mecanismos para lograr que sus objetos sean vinculantes. El primero se refiere las instancias de consulta que obligan a los municipios a someter sus expectativas de crecimiento al juicio de los niveles superiores de gobierno; el segundo radica en la implementación de leyes provinciales para proteger los componentes territoriales metropolitanos (los frentes fluviales, el pie de monte, la red vial primaria, el suelo periurbano, etc.); y el tercero se basa en el apoyo técnico a los municipios para que redacten sus propios planes urbanos, garantizando, de este modo, que incorporen las "cuestiones metropolitanas" en su ADN.

(3) Si bien en el proceso de planeamiento metropolitano se contemplan escenarios y costos de inversión, la gestión supralocal no está atada a un programa de actuaciones, sino a un elenco de obras concretas. No se trata de empezar por el final, sino de mostrar que las políticas metropolitanas son "reales". Así, la incipiente consolidación institucional convierte al programa de acciones -la agenda común plurimunicipal- en un objetivo a mediano plazo. Mientras tanto, el proceso de planeamiento se apoya en diversas fórmulas de participación: talleres y encuestas ciudadanas, y mesas de funcionarios y técnicos. El papel protagonista de estos últimos ha sido clave para promover la consciencia de "lo metropolitano" desde una mirada urbanística y geográfica; mientras que la participación de los funcionarios se ha enfocado más en los aspectos políticos para desentrañar la sutil diferencia entre "cooperación" y "coordinación". 
Sin la pretensión de fundar un nuevo tipo o estilo de planeamiento, o de agotar el conocimiento acerca de los casos, los resultados de la comparación pretenden esbozar un estilo tecnológico que, por su singular conjugación de formas y estrategias, hemos llamado "heterodoxo". Esta denominación es deudora del modelo de planeamiento no-euclidiano que proponía John Friedmann a mediados de los noventas, cuyo planteo procuraba ser superador del planeamiento tradicional -ortodoxo- propio del modernismo ingenieril, y vigente desde los albores de la revolución industrial. El planeamiento ortodoxo, decía Friedmann (1993), es aquel que pretende ser neutral dirigiendo sus objetivos a la "humanidad"; que pretende ser científico limitándose a las recetas, estándares e indicadores enlatados; que intenta ser operativo limitando su actividad principalmente en la producción de documentos elaborados, en general, al margen del escrutinio público; y que anhela ser democrático abogando por una adhesión estricta al código urbano, a la neutralidad afectiva y la tecnocracia apolítica -ubicándose cómodamente en el centro. El planeamiento no-euclidiano, en cambio, opera en tiempo real, emprendiendo, tomando riesgos y asumiendo públicamente las responsabilidades. Ata el conocimiento y la acción para superar la resistencia al cambio, fijando las exigencias de implementación adecuadas a cada situación, y abogando por una gestión basada en las transacciones cara a cara -el diálogo- entre los planificadores y la población. Así, el nuevo modelo pretende operar en las escalas de la representación más directa, nutriéndose del aprendizaje social basado en soluciones flexibles, en la retroalimentación crítica y en la memoria institucional.

A partir de los planteos de Friedmann, la definición de planeamiento heterodoxo que se propone profundiza en las principales discusiones teóricas de los últimos veinte años, en particular, de aquellas surgidas del "giro espacial" y del "giro hacia el sur".

Desde el punto de vista espacial, el enfoque no-euclidiano pone en tela de juicio el carácter autorreferencial de la territorialidad formal -euclidiana- del Estado frente a las dinámicas territoriales con geometría variable. Como se evidencia en los dos casos, el planeamiento heterodoxo no reconoce jerarquías preestablecidas. En cambio, intenta superar las escalas cosificadas que obstaculizan los procesos políticos y (de)limitan la agencia práctica como resultado de la organización y fijación previa del poder. Es en este sentido que el planeamiento heterodoxo se apoya en una "ontología escalar plana y horizontal", tal como lo planteaba Sallie Marston (2005) siguiendo las lecciones de DeLanda, Deleuze y Latour. Si algo muestran los dinámicos territorios metropolitanos es que el devenir de los espacios es contingente, y que la horizontalidad provee nuevas puertas de entrada a lo político, reforzando las conexiones entre los actores sociales y las escalas, donde "arriba" y "abajo" siempre es relativo.

Además, profundizar en los aspectos no-euclidianos implica marcar una diferencia en cuanto a lo que significa planificar "desde" y "la" periferia. Es en este sentido, que la noción de planeamiento heterodoxo incorpora la teoría del Sur Global (véase, Connell, 2014; Watson, 2016; Roy, 2015; y Miraftab, 2017, entre otros). Más allá de las connotaciones geográficas, "Sur Global" es una estrategia conceptual que implica comprender los procesos del colonialismo, el poscolonialismo, el imperialismo y el capitalismo, a través de una nueva epistemología de la planificación. En este sentido, permite revisar conceptos introducidos por el planeamiento ortodoxo (por ejemplo "subdesarrollo" o "informalidad") como parte de un proyecto general de descolonización del pensamiento social.

Para finalizar, planteamos algunas cuestiones abiertas a la indagación de futuras investigaciones. Por un lado, se debería evaluar cuál ha sido el efecto de las políticas metropolitanas desarrolladas en el contexto de cada modelo institucional y, por otro, hasta qué punto el planeamiento metropolitano heterodoxo cristaliza en un nuevo nivel administrativo, haciendo que la dependencia técnica y presupuestaria se torne estructural. 


\section{BIBLIOGRAFIA}

BRINDLEY, T., RYDIN, Y., \& STOKER, G. (1996). Remaking Plannig: the politics of urban change. London: Routledge.

CONNELL, R. (2014). Using southern theory: Decolonizing social thought in theory, research and application. Planning Theory, 13(2), 210-223.

EUROPEAN COMISSION (1997). The EU compendium of spatial planning systems and policies. Luxembourg ;Lanham MD: Office for Official Publications of the European Communities; Bernan Associates [distributor].

ELINBAUM, P., \& GALLAND, D. (2016). Analysing Contemporary Metropolitan Spatial Plans in Europe Through Their Institutional Context, Instrumental Content and Planning Process. European Planning Studies, 24(1).

ELINBAUM, P. (2016). Planes fuera del sistema. Instrumentos ad hoc para la ordenación y gestión de las áreas urbanas plurimunicipales. Eure, 42(127).

ELINBAUM, P. (2018). Planning systems in Latin America. The case of Argentina. Special Edition in Latin America. DisP - The Planning Review - Taylor and Francis.

FALUDI, A., \& VAN DER VALK, A. (1994). Rule and order: Dutch planning doctrine in the twentieth century. Dordrecht; Boston: Kluwer Academic Publishers.

FRIEDMANN, J. (1993). Toward a Non-Euclidian Mode of Planning. Journal of the American Planning Association, 59(4), 482-485. https://doi.org/10.1080/01944369308975902

GALLAND, D., \& ELINBAUM, P. (2018). A "Field" Under Construction: The State of Planning in Latin America and the Southern Turn in Planning. DisP - The Planning Review, 54(1), 18-24.

JANIN RIVOLIN, U. (2008). Conforming and performing planning systems in Europe: An unbearable cohabitation. Planning, Practice \& Research, 23(2), 167-186.

MARSTON, S. A., JONES, J. P., \& WOODWARD, K. (2005). Human geography without scale. Transactions of the Institute of British Geographers, 30(4), 416-432.

MEIJERS, E., ROMEIN, A., \& HOPPENBROUWER, E. (2003). Planning polycentric urban regions in North West Europe : value, feasibility and design. Delft: DUP Science.

MIRAFTAB, F. (2017). Insurgent practices and decolo- nization of future(s). In M. Gunder, A. Madanipour, \& V. Watson (Eds.). The Routledge handbook of plan- ning theory. London and New York: Routledge.

NEWMAN, P., \& THORNLEY, A. (1996). Urban planning in Europe : international competition, national systems, and planning projects. London; New York: Routledge.

ROODBOL-MEKKES, P. (2012). The Netherlands spatial planning doctrine in disarray in the 21st century. Environment and Planning $A$.

ROY, A. (2015). Who's afraid of postcolonial theory? In- ternational Journal of Urban and Regional Research, 40(1), 200-209. 
WATSON, V. (2016). Shifting Approaches to Planning Theory : Global North and South. Urban Planning, 1(4), 32-41. https://doi.org/10.17645/up.v1i4.727 